\title{
IDADE ÓSSEA EM CRIANÇAS COM PARALISIA CEREBRAL
}

\author{
EdUARDO MARCONDES * \\ MARIA IRMina VALENTE ** \\ Francisco F. De Fiore *** \\ Antonio da Silva Coelho Neto ****
}

Crianças portadoras de paralisia cerebral (PC) apresentam, do ponto de vista clínico, variados graus de retardo do desenvolvimento neuro-psico-motor associados a também variados graus de comprometimento do crescimento físico e do estado nutritivo. Em alguns casos a predominância do retardo psíquico e do crescimento físico sôbre manifestações neurológicas motoras obriga o estabelecimento de diagnóstico diferencial entre PC e outras formas de retardo; entre estas últimas sobressai o hipotiroidismo exigindo a realiżação de exames subsidiários adequados como seja, entre outros, a determinação da idade óssea (IO).

Em trabalhos anteriores ${ }^{6,7}$ verificou-se a existência de um atraso da IO em relação à idade cronológica (IC) em crianças oriundas de meio sócio-econômico baixo, porém compensado se a IO fosse relacionada ao pêso das crianças, o que mostra ser o atraso da IO proporcional à deficiência somática geral das referidas crianças. A hipótese de trabalho estabelecida para a presente investigação é que os eventuais atrasos das IO verificados em crianças portadoras de $\mathrm{PC}$ em relação à IC são também meros componentes do atraso somático geral que tais crianças costumam apresentar e, portanto, a IO deve ser proporcional ao crescimento físico (pêso e altura) e não ao tempo de vida. Se isto fôr verdade, o atraso da IO, como dado isolado, em crianças com PC, carece de importância para efeito de diagnóstico de condições mórbidas outras que não a própria PC.

\section{MATERIAL E MÉTODOS}

Foram estudadas 30 crianças com PC. Os dados de identificação e a diagnóstico neurológico são apresentados no quadro 1. A IO foi determinada de acôrdo com Greulich e Pyle ${ }^{3}$. Em algumas crianças foram feitas determinações do iôdo

Trabalho realizado na Clínica Pediátrica (Prof. Substituto Eduardo Marcondes), Clínica Neurológica (Prof. Adherbal Tolosa) e 1. Clinica Médica (Prof. A. B. Ulhoa Cintra) da Faculdade de Medicina da Universidade de São Paulo: * Professor Catedrático Substituto da Clínica Pediátrica; ** Assistente do Grupo de Neuropediatria da Clinica Neurológica; *** Assistente, Chefe do Ambulatórío da Clínica Pediátrica; **** Assistente do Grupo de Nutrição da $1^{*}$ Clínica Médica.

Nota dos autores - Agradecemos a colaboração prestada pelo Dr. Afonso Vitule Filho, do Serviço de Radiologia do Hospital das Clínicas. 


\begin{tabular}{|c|c|c|c|c|c|c|c|}
\hline Caso & Prontuário & Sexo & $\begin{array}{c}\text { Diagnóstico neuro- } \\
\text { lógico }\end{array}$ & $\begin{array}{c}I C \\
(m e- \\
\text { ses) }\end{array}$ & $\begin{array}{l}\text { Pêso } \\
(100 \mathrm{~g})\end{array}$ & $\begin{array}{c}\text { Altura } \\
(\mathrm{cm})\end{array}$ & $\begin{array}{l}\text { IO } \\
\text { (me- } \\
\text { ses) }\end{array}$ \\
\hline 1 & P-19.405 & $\mathbf{M}$ & Hemiparesia esquerda & 18 & 85 & 73 & 6 \\
\hline 2 & $\mathrm{~N}-42.929$ & $\mathbf{M}$ & Dupla hemiplegia & 25 & 85 & 76 & 6 \\
\hline 3 & $\mathrm{~N}-46.251$ & $\mathbf{M}$ & Dupla hemiplegia & 36 & 99 & 83 & 15 \\
\hline 4 & $\mathrm{~N}-45.331$ & $\mathbf{M}$ & Dupla hemiparesia & 48 & 128 & 95 & 30 \\
\hline 5 & P-14-595 & $\mathbf{M}$ & Dupla hemiplegia & 49 & 142 & 99 & 30 \\
\hline 6 & $\mathrm{~N}-38.153$ & $\mathbf{M}$ & Dupla hemiplegia & 51 & 148 & 100 & 48 \\
\hline 7 & $P-19.3 C 8$ & $\mathbf{M}$ & Retardo psico-motor & 52 & 136 & 94 & 18 \\
\hline 8 & $\mathrm{~N}-48.44 \overline{4}$ & $\mathbf{M}$ & Dupla hemiparesia & 63 & 111 & 89 & 30 \\
\hline 9 & $N-52-427$ & $\mathbf{M}$ & Dupla hemiplegia & 64 & 115 & 93 & 30 \\
\hline 10 & $N-40-603$ & $\mathbf{M}$ & Diplegia cerebral & 66 & 181 & 103 & 36 \\
\hline 11 & $P-16.378$ & $\mathbf{M}$ & Retardo psíquico & 66 & 121 & 94 & 12 \\
\hline 12 & P-19.145 & M & Não especificado & 66 & 144 & 99 & 24 \\
\hline 13 & P-19.362 & $\mathbf{M}$ & Dupla hemiplegia & 80 & 123 & 99 & 38 \\
\hline 14 & P-20.038 & $\mathbf{M}$ & Não especificado & 90 & 115 & 96 & 42 \\
\hline 15 & $P-20.400$ & $\mathbf{M}$ & Dupla hemiparesia & 96 & 131 & 97 & 66 \\
\hline 16 & P-19.374 & $\mathbf{F}$ & Dupla hemiparesia & 12 & 77 & 68 & 12 \\
\hline 17 & P-19.307 & $\mathbf{F}$ & Dupla hemiparesia & 17 & 88 & 73 & 36 \\
\hline 18 & P-19.222 & $\mathbf{F}$ & Dupla hemiplegia & 21 & 92 & 76 & 6 \\
\hline 19 & N-43.255 & $\mathrm{F}$ & Dupla hemiplegia & 24 & 79 & 76 & 24 \\
\hline 20 & $\mathrm{~N}-46.248$ & $\mathrm{~F}$ & Dupla hemiplegia & 24 & 124 & 80 & 15 \\
\hline 21 & $\mathrm{~N}-45.853$ & $\mathbf{F}$ & Dupla hemiplegia & 25 & 97 & 78 & 36 \\
\hline 22 & $\mathrm{~N}-47.246$ & $\mathrm{~F}$ & Hemiparesia direita & 27 & 92 & 79 & 32 \\
\hline 23 & N-46.374 & $\mathbf{F}$ & Retardo psico-motor & 27 & 79 & 73 & 12 \\
\hline 24 & $\mathrm{~N}-46.241$ & $\mathbf{F}$ & Diplegia cerebral & 29 & 100 & 82 & 12 \\
\hline 25 & $\mathrm{~N}-45.406$ & $\mathbf{F}$ & Retardo psiquico & 29 & 102 & 78 & 12 \\
\hline 26 & $\mathrm{~N}-40.240$ & $\mathbf{F}$ & Dupla hemiplegia & 30 & 106 & 84 & 32 \\
\hline 27 & $\mathrm{~N}-47.163$ & $\mathbf{F}$ & Ataxia & 32 & 88 & 82 & 32 \\
\hline 28 & $P-19.594$ & $\mathbf{F}$ & Dupla hemiparesia & 34 & 106 & 85 & 18 \\
\hline 29 & P-20.144 & $\mathbf{F}$ & Não especificado & 50 & 115 & 96 & 36 \\
\hline 30 & P-19.573 & $\mathbf{F}$ & Retardo psíquico & 50 & 118 & 89 & 50 \\
\hline
\end{tabular}

Quadro 1 - Prontuário $(P=$ Clinica Pediátrica e $N=$ Clinica Neurológica), sexo, diagnóstico neurológico, idade cronológica (IC), pêso, altura $e$ idade óssea (IO) dos 30 casos estudados. 
ligado à proteina (P.B.I.), colesterol, cálcio, fósforo inorgânico e atividade da fosfatase alcalina bem coom exame radiológico da bacia para pesquisa de sinais de disgenesia epifisária.

O estudo da IO e suas relações com a IC, altura e pêso foi feito gråficamente tomando-se sempre como ordenada a IO e como abscissa sucessivamente a IC, altura e pêso. Dos diagramas assim obtidos obtiveram-se, sempre que possivel, retas de regressão. O têrmo de comparação é representado por uma reta que traduz a regressão existente para os dados em questão obtidos num grupo contrôle de crianças normais ${ }^{8}$.

\section{RESULTADOS}

Os valores de IC, pêso, altura e IO relativos aos 30 casos estudados são apresentados no quadro 1 . Os resultados das determinações de P.B.I., colesterol, cálcio, fósforo inorgânico e atividade da fosfatase alcalina e da pesquisa de sinais de disgenesia epifisária em alguns dos casos estudados são apresentados no quadro 2 . As relaçōes entre IO e IC, altura e pêso são apresentadas nos gráficos 1 e 2 , respectivamente para o sexo masculino e feminino. O estudo em separado conforme o sexo é uma decorrência do fato das retas de regressão obtidas para o grupo contrôle serem diferentes para cada um dos sexos.

\begin{tabular}{|c|c|c|c|c|c|c|c|c|}
\hline Caso & Prontuário & Sexo & $\begin{array}{c}\text { Sinais de } \\
\text { disgene- } \\
\text { sia epi- } \\
\text { fisária }\end{array}$ & $\begin{array}{c}P B I \\
\mathrm{mcg} \%\end{array}$ & $\begin{array}{c}\text { Colest. } \\
m g \%\end{array}$ & $\begin{array}{c}C a \\
m g \%\end{array}$ & $\begin{array}{c}P \\
m g \%\end{array}$ & $\begin{array}{l}A F A \\
u . K A\end{array}$ \\
\hline 1 & P-19.405 & $\mathbf{M}$ & Não & 11,8 & 185 & 9,9 & 5,7 & 15,7 \\
\hline 5 & P-14.545 & M & Não & 9,9 & 155 & 9,9 & 6,2 & 11,0 \\
\hline 7 & P-19.308 & M & Não & 6,2 & 149 & 9,8 & 4,9 & 22,0 \\
\hline 11 & P-16.378 & M & Sim & 6,8 & 179 & - & - & - \\
\hline 13 & P-19.362 & M & Não & 7,0 & 275 & 9,8 & 5,1 & 17,2 \\
\hline 16 & P-19.574 & $\mathbf{F}$ & Nāo & 8,8 & 167 & 10,2 & 5,2 & 6,8 \\
\hline 17 & P-19.307 & $\mathbf{F}$ & - & 9,4 & 132 & 9,9 & 4,9 & 15,9 \\
\hline 18 & P-19.222 & $\mathbf{F}$ & Não & 5,7 & 198 & 10,3 & 6,2 & 6,9 \\
\hline 28 & P-19.594 & $\mathbf{F}$ & Não & 6,3 & 173 & 10,9 & 5,3 & 21,1 \\
\hline 30 & P-19.573 & $F$ & - & 10,7 & 275 & 11,9 & 2,1 & 25,7 \\
\hline
\end{tabular}

Quadro 2 - Exame radiológico da bacia, dosagens de iôdo ligado à proteina (PBI), de colesterol, de cálcio ( $\mathrm{Ca}$ ), de fósforo inorganico $(P)$ e da atividade da fosfatase alcalina (AFA) em 10 casos.

A inspeção dos gráficos 1 e 2 mostra que para o sexo masculino é possivel estabelecer regressão entre IO e IC e entre IO e altura e que para o sexo feminino só é possivel estabelecer regressão entre IO e IC. De fato, o valor de F para as retas correspondentes à distribuição dos pontos relativos à IO e pêso para o sexo masculino e à IO e altura como tamiém à IO e pêso para o sexo feminino mostra que tais retas não são significantes. Os elementos característicos das retas obtidas são apresentadas no quadro 3. 


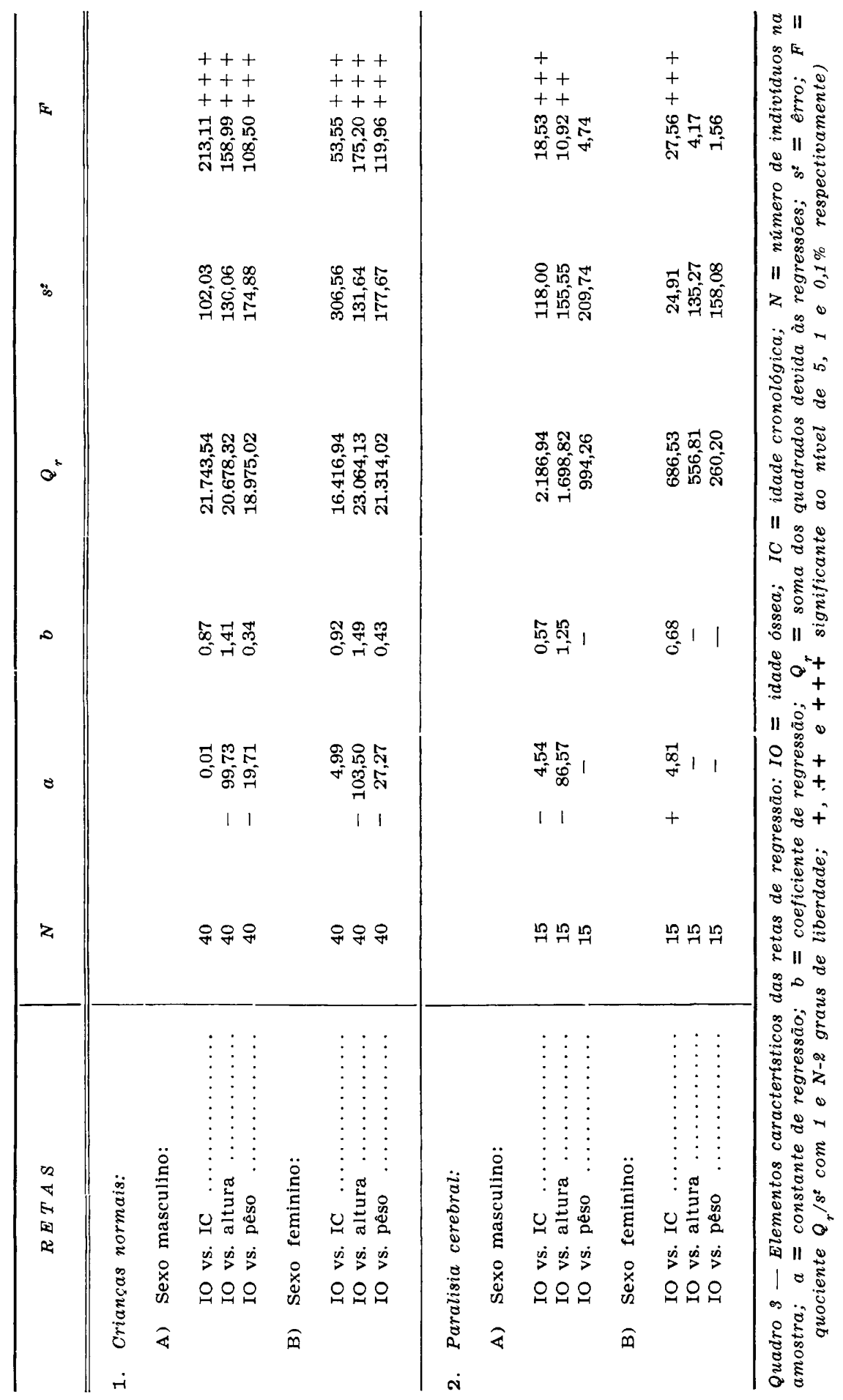




\section{COMENTARIOS}

É reconhecida a influência da nutrição sôbre a $I^{1}$ 1, 2, 6, 7, 11 bem como o comprometimento do estado nutritivo de crianças portadoras de PC. Legitimo, pois, o interêsse pelo relacionamento da IO com o estado nutritivo de crianças encefalopatas. Na casuistica do presente trabalho basta a comparação do pêso e da IA (quadro 1) para comprovar o precário estado nutritivo de quase todos os casos estudados.

Os dados para o sexo masculino (gráfico 1) mostram que no relacionamento entre a IO e a IC ocorre nítido atraso daquela em relação a esta. Assim é que sòmente um caso apresenta IO comparável à IC. O mesmo não se verifica quando se relaciona a IO com a altura e, principalmente, quando a relação estabelecida é entre a IO e o pêso: aqui se verifica que as crianças portadoras de $\mathrm{PC}$ têm IO superior à do grupo contrôle pois dos 15 casos estudados, 9 localizam-se acima da reta normal. É de se notar que embora a IO em relação ao pêso possa ser considerada satisfatória, a distribuição dos pontos não permite concluir pela existência de regressão entre IO e pêso.

Em relação ao sexo feminino (gráfico 2) nota-se, de início, nítida diferença no comportamento da IO em relação à IC em função do sexo da criança, pois a distribuição dos pontos para os casos de sexo feminino permite supor que os pacientes desse sexo resistam mais aos fatores determinantes do atraso do que os pacientes do sexo masculino. No relacionamento da IO com a altura e o pêso, verifica-se comportamento semelhante ao observado para os pacientes de sexo masculino, isto é, evidente recuperação da IO quando comparada com os referidos parâmetros. A distribuição dos pontos, entretanto, não é adequada para o estabelecimento de boa regressão.

Ora, em trabalho anterior ${ }^{7}$ verificou-se que crianças desnutridas apresentam IO atrasadas em relação à IC, atraso compensado quando a IO era relacionada ao pêso, porém com possibilidade de estabelecer regressão entre a IO e o pêso, o que não ocorreu neste trabalho. É possivel que a PC determine também um atraso na IO quando relacionada à IC compensado quando a IO é avaliada em relação ao pêso e altura, mas, ao contrário das crianças desnutridas há evidentemente na PC um fator perturbador do desenvolvimento da IO, talvez ao nível de seu próprio orgão efetor. Assim sendo, a desnutrição que acompanha a PC seria responsável pelo atraso e a PC por si mesma responsável pela inexistência de regressão entre IO e pêso para ambos os sexos e entre IO e altura para o sexo feminino.

Com a finalidade de comprovar que o atraso da IO não corresponderia a uma insuficiência tireóidea, foram feitas determinações especiais em 10 casos (quadro 2): as dosagens de colesterol, cálcio, fósforo e atividade da fosfatase alcalina resultaram pràticamente normais em todos os casos. Quanto ao P.B.I., os resultados obtidos afastam o diagnóstico de hipotireóidismo. Em 8 pacientes foi feito estudo radiológico da bacia para pesquisa de disgenesia epifisária, com resultado positivo em um caso que, aliás, apresentava P.B.L. normal. A julgar por êstes resultados, a coexistência de hipotireoidismo e PC é uma situação de exceção. 


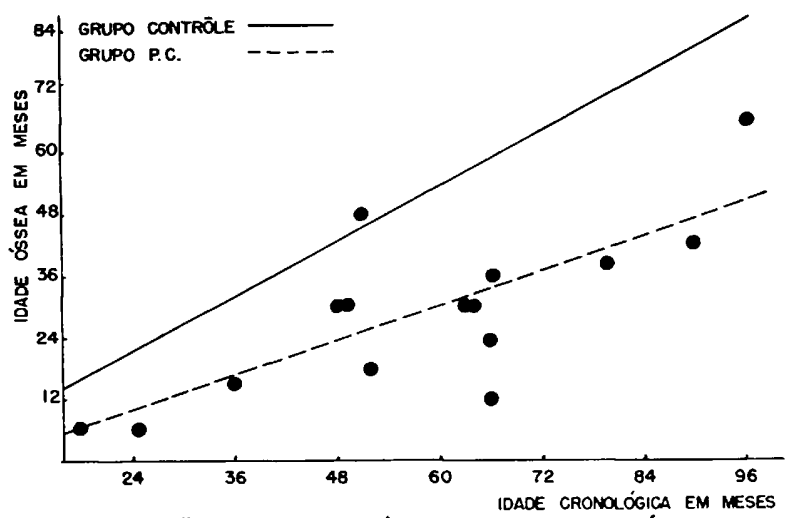

RELACÄO ENTRE IDADE ÓSSEA E IDADE CRONOLÓGICA

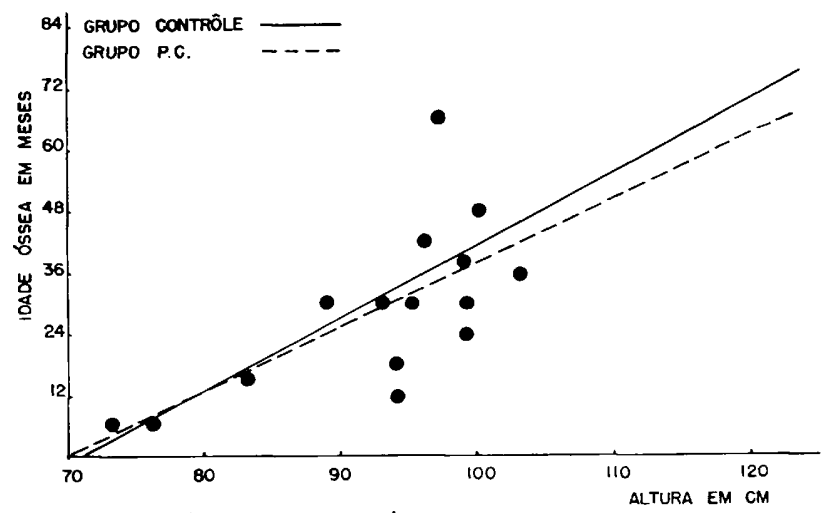

RELACĀO ENTRE IDADE ÓSSEA E ALTURA

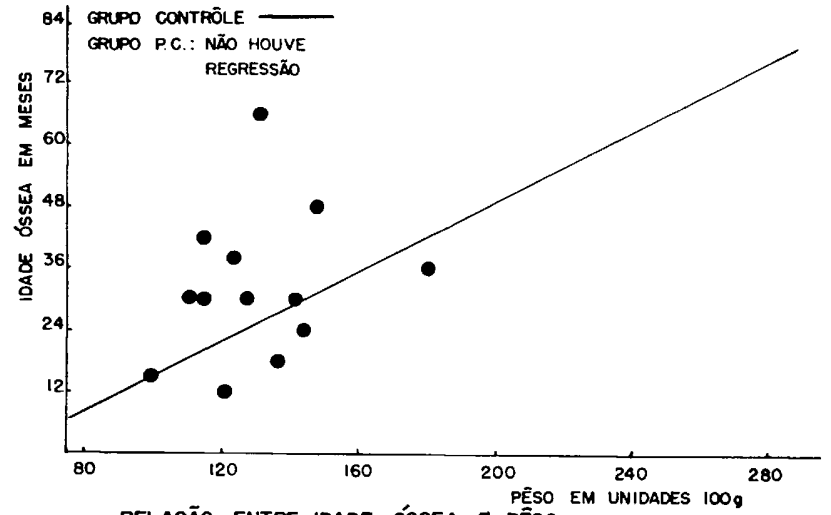

RELACĀo ENTRE IDADE ÓSSEA E PÊsO

Gráfico 1 - Relações referentes ao sexo masculino. 

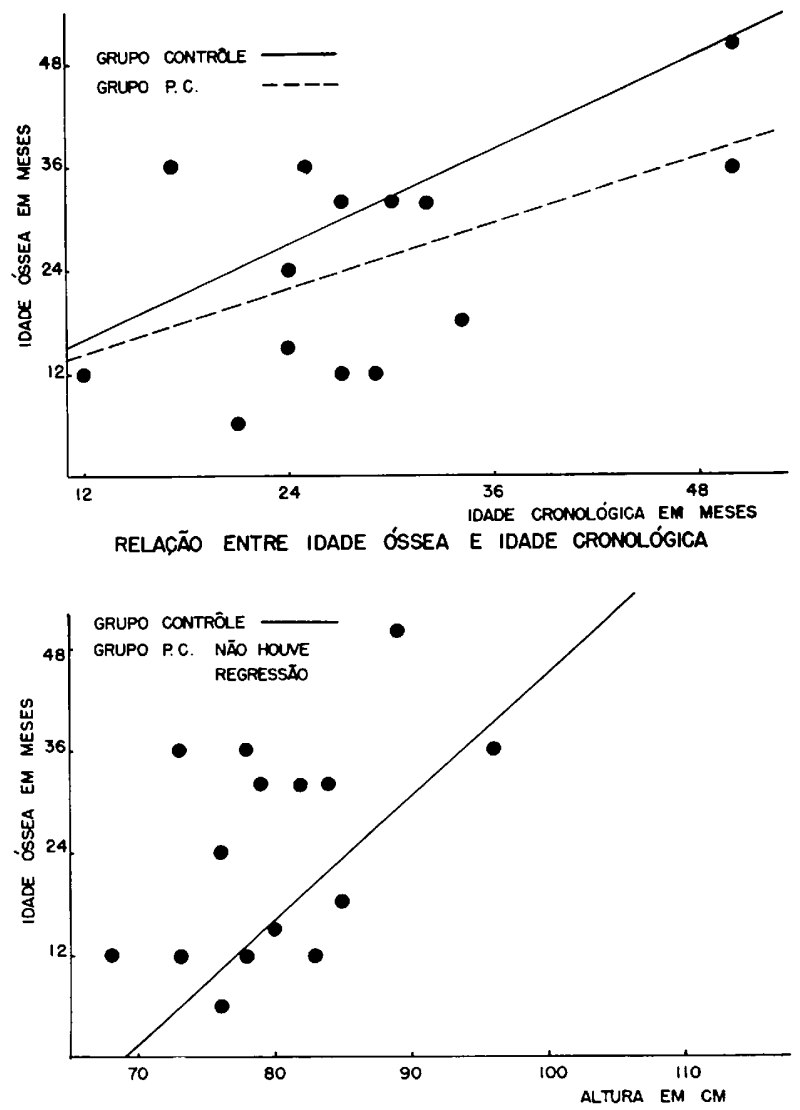

RELACÄO ENTRE IDADE ÓSSEA E ALTURA

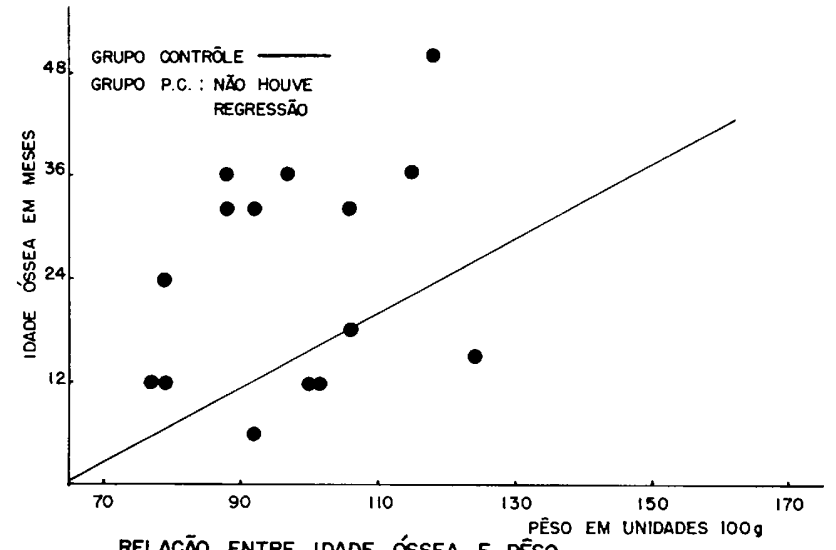

RELAÇĀO ENTRE IDADE ÓSSEA E PÉso

Gráfico 2 - Relações referentes ao sexo feminino. 
$O$ fato dos pacientes encefalopatas de sexo feminino resistirem melhor aos fatores determinantes do atraso da IO, é mais uma evidência da superioridade do sexo feminino em relação ao masculino na resistência aos fatores ambientais adversos 4, 5, 9, 10 .

\section{SUMARIO}

Os autores estudam a idade óssea de 30 crianças portadoras de paralisia cerebral. Verificaram haver atraso da idade óssea em relação à idade cronológica (mais evidente no sexo masculino), o que não impediu que continuasse a existir regressão entre os dois dados. Relacionando a idade óssea à altura e o pêso, verificaram que as crianças portadoras de paralisia cerebral têm idade óssea superior à esperada, mas só foi possivel estabelecer regressão entre idade óssea e altura em crianças do sexo masculino. Estudos de função tireóidea em 10 casos mostraram normalidade em praticamente todos êles. Os dados sugerem: 1) atraso da idade óssea de crianças com paralisia cerebral, proporcional ao atraso somático geral, possìvelmente em função da desnutrição que tais crianças apresentam; 2) perturbação das relações de proporcionalidade entre idade óssea e pêso para o sexo masculino e entre idade óssea e pêso e idade óssea e altura para o sexo feminino, possivelmente na dep̣endência da própria paralisia cerebral; 3) a ação desorganizadora da para. lisia cerebral não deve depender de um componente de hipotireóidismo; 4) as alterações verificadas são mais intensas no sexo masculino.

\section{SUMMARY}

\section{Skeletal age in children with cerebral palsy}

The skeletal age of thirty children with cerebral palsy was studied and related to the chronologic age, height and weight. In 10 cases the thyroid function was evaluated. The results allow the following suggestions: 1) the skeletal age and the somatic development were proportionally delayed and both probably related to the poor nutritional state of the children; 2) the proportionality between skeletal age and weight among the male children, and between the skeletal age and weight as well as height among the female children, were altered; 3 ) the disorganizing action of the cerebral palsy should not depend on hypothyroidism; 4) the noted alterations are more conspicuous among the male children.

\section{REFERENCIAS}

1. ABBot, O. D.; TOWSEND, R. O.; FRENCH, R. B. \& AHMANN, C. F. Carpal and epiphyseal development: another index of nutritional status of rural school children. Am. J. Dis. Child. 79:69-81, 1950. 2. BRUSA, P. \& MENGHI, P. - Sulla valutazione dell'eta scheletrica nei bambini distrofici. Minerva pediat. 6: 649-651, 1954. 3. GREULICH, W. W. \& PYLE, S. I. - Radiographic Atlas of Skeletal Development of the Hand and Wrist. Stanford Univ. Press, Stanford, 1950.4. 
GREULICH, W. W. - The growth and development status of guamanian schol children in 1947. Am. J. Phys. Anthrop. 9:55-70, 1951. 5. GREULICH, W. W.; CRISMON, C. S. \& TURNER, M. F. - The physical growth and development of children who survived the atomic bombing of Hirochima and Nagasaki. J. Ped. 43:121-145, 1953. 6. MACHADO, E. M.; ChAMMAS, F.; COElHO, H. da S.; SIlVA, J. A. da; GOUVEIA, F. P. de; NEVES Filho, F. de P. \& CONCEIÇAO, J. A. N. - Estudo sôbre idade óssea, cálcio, fósforo e atividade da fosfatase alcalina em crianças em meio sócio-econômico inferior: resultados em lactentes. Rev. Hosp. Clín. 15:81-91, 1960. 7. MACHADO, E. M.; CHAMMAS, F.; NEVES Filho, F. de P.; COElHo, H. da S. \& SILVA, J. A. da - Estudo sôbre idade óssea, cálcio, fósforo e atividade da fosfatase alcalina em crianças em meio sócio-econômico inferior: resultados em préescolares. Rev. Paulista Med. 58:163-172, 1961. 8. MARCONDES, E. - Dados não publicados. 9. PHILlIPS, H. T. - Some social and ethnic variations in the physique of South African nursery school children. Arch. Dis. Child. 28:226-231, 1953. 10. SALBER, E. J. - The effect of sex, birth rank and birth weight on growth in the first year of life. Human Biology, 29:194-213, 1957. 11. TROWELL, H. C.; DAVIES, J. N. P. \& DEAN, R. F. A. - Kwashiorkor: clinical picture, pathology and differential diagnosis. Brit. M. J. 2:798-801, 1952.

Clinica Pediátrica. Faculdade de Medicina da Universidade de São Paulo Caixa Postal 22067 - São Paula, SP, Brasil. 